Cahiers de géographie du Québec

\title{
Protection et gestion dans les parcs nationaux canadiens et états-uniens.
}

\author{
Quelques enjeux actuels
}

\section{Some Issues Concerning National-Park Protection and Management in North America (Canada and United States)}

\section{Stéphane Héritier et Caroline Moumaneix}

Volume 51, numéro 143, septembre 2007

URI : https://id.erudit.org/iderudit/016598ar

DOI : https://doi.org/10.7202/016598ar

Aller au sommaire du numéro

Éditeur(s)

Département de géographie de l'Université Laval

ISSN

0007-9766 (imprimé)

1708-8968 (numérique)

Découvrir la revue

Citer cet article

Héritier, S. \& Moumaneix, C. (2007). Protection et gestion dans les parcs nationaux canadiens et états-uniens. Quelques enjeux actuels. Cahiers de géographie du Québec, 51(143), 155-176. https://doi.org/10.7202/016598ar

\section{Résumé de l'article}

Depuis 1872 (création du parc national de Yellowstone) pour les États-Unis et 1885 (création du parc national de Banff) pour le Canada, les deux grands États d'Amérique du Nord ont, chacun, développé un système de parcs nationaux comptant aujourd'hui 100 parcs et accueillant près de 100 millions de visiteurs par an. Ils participent ainsi d'un vaste système touristique - organisé à différentes échelles - qui s'appuie sur la valorisation de la nature, des phénomènes géologiques et géomorphologiques, mais également sur la médiation des rapports entre les sociétés passées et cette nature (à travers des pratiques d'interprétation). Territoires convoités par des activités concurrentes, les parcs sont, depuis l'origine, un sujet d'enjeux particulièrement intenses entre différentes composantes de la société qui, chacune, revendiquent des usages spécifiques. Ces tensions contribuent à produire, à imposer ou à légitimer, selon les cas, des réglementations destinées à réguler les pratiques et les usages de l'espace dans les parcs (stratégies spatiales, stratégies de gestion ou de contrôle des flux, etc.) 


\section{Protection et gestion dans les parcs nationaux canadiens et états-uniens. Quelques enjeux actuels ${ }^{1}$}

\author{
Some Issues Concerning National-Park \\ Protection and Management in North \\ America (Canada and United States)
}

\section{Résumé}

Depuis 1872 (création du parc national de Yellowstone) pour les États-Unis et 1885 (création du parc national de Banff) pour le Canada, les deux grands États d'Amérique du Nord ont, chacun, développé un système de parcs nationaux comptant aujourd'hui 100 parcs et accueillant près de 100 millions de visiteurs par an. Ils participent ainsi d'un vaste système touristique - organisé à différentes échelles - qui s'appuie sur la valorisation de la nature, des phénomènes géologiques et géomorphologiques, mais également sur la médiation des rapports entre les sociétés passées et cette nature (à travers des pratiques d'interprétation). Territoires convoités par des activités concurrentes, les parcs sont, depuis l'origine, un sujet d'enjeux particulièrement intenses entre différentes composantes de la société qui, chacune, revendiquent des usages spécifiques. Ces tensions contribuent à produire, à imposer ou à légitimer, selon les cas, des réglementations destinées à réguler les pratiques et les usages de l'espace dans les parcs (stratégies spatiales, stratégies de gestion ou de contrôle des flux, etc.)

\section{Mots-clés}

Parcs nationaux, tourisme, gestion, Amérique du Nord, relation nature-société, protection de l'environnement

\author{
Stéphane HÉRITIER \\ Université Jean Monnet \\ step_heritier@hotmail.com \\ Caroline MOUMANEIX \\ Université de Paris IV-Sorbonne \\ caromoums@aol.com
}

\section{Abstract}

Since the creation of Yellowstone National Park in 1872 by the United States and of the Banff Hot Springs Reservation (future Banff National Park) in 1885 by Canada, the two main large North American states have set up a network of 100 national parks, attracting close to 100 million visitors per year. Parks are crucial to a large tourist system, organized at different scales, which relies on the highlighting of natural, geological or geomorphological phenomena as well as on the ties between past societies and today's nature (interpretation programs). Territories coveted by competing activities, parks are the focus of intense issues involving different components of society which claim them for specific uses. These tensions contribute to producing, imposing or legitimizing rules to regulate the practices and uses of space in the parks (spatial strategies, management or control of flows, etc.).

\section{Keywords}

North America, national parks, tourism, nature management, man and nature relationship, environmental protection 
Avec l'établissement du parc de Yellowstone en 1872, les États-Unis créaient une nouvelle idée, celle du parc national. Si des opérations de mise en réserve avaient déjà vu le jour (par exemple aux États-Unis avec la réserve Arkansas Hot Springs en 1832 ou en France avec la réserve artistique de Fontainebleau en 1853) (Dubois et al., 2005), aucune encore n'avait atteint l'ampleur du parc national de Yellowstone, protégeant un vaste espace de hauts plateaux dans les Rocheuses américaines, aux confins ouest de l'État du Wyoming. Cette création s'inscrivait dans un contexte particulier, celui de la progression vers l'ouest des colons attirés par les terres à bon marché et le désir d'une vie nouvelle, dans un mouvement soutenu par la mythologie politique de la Destinée manifeste - manifest destiny - et dans une période de croissance démographique et urbaine, qui faisait craindre la dégradation rapide des ressources... et de la nature en général.

Dans ce contexte, l'ouvrage de George Perkins Marsh (1864) intitulé Man and Nature, or, Physical Geography as Modified by Human Action, sonna comme une alerte particulièrement troublante quant à l'état de la nature. Ce diplomate américain trouva, lors de ses missions en Europe, des études montrant que les sociétés humaines faisaient de la nature - on dirait aujourd'hui de l'environnement - un usage pouvant provoquer une multitude de dégradations d'intensité et d'ampleur variables, rendant nécessaire les mesures de protection des milieux de vie. Cette réflexion s'inscrivait dans une période marquée par l'affirmation du transcendantalisme, à l'origine d'une nouvelle conception de la nature et des relations de l'être humain avec celle-ci (Nash, 1982; Oelschlaeger, 1991). Elle se diffusa autant aux États-Unis qu'au Canada ${ }^{2}$. Les œuvres majeures de Whitman, d'Emerson et de Thoreau eurent un écho important parmi les élites intellectuelles et politiques, tant états-uniennes que canadiennes. On comprend mieux que, dans des conditions voisines de celles des États-Unis - celles de la conquête des territoires de l'ouest, le Canada ait créé sa première réserve fédérale à Banff, dans le sillage de la création de la voie ferrée transcontinentale, en 1885 (Glazebrook, 1964 ; Lothian, 1987).

L'originalité du service canadien des parcs et du National Park Service états-unien tient en premier lieu à leur ancienneté. Ces pays disposent de la plus longue et de la plus grande expérience en termes de protection de l'espace et de gestion des aires protégées (respectivement 122 ans et 135 ans d'existence en 2007). L'histoire de ces deux services, chargés de gérer les parcs fédéraux auxquels nous consacrons cette étude, n'est en rien linéaire et elle a été caractérisée par des phases de mise en valeur contradictoires et conflictuelles (Sellars, 1997; Bella, 1987; Dearden et Rollins, 1993) qui sont responsables d'une partie des problèmes actuels. Depuis les années 19601970, les parcs nationaux ont globalement été caractérisés par une augmentation considérable de la fréquentation qui a conduit dans certains cas à des phénomènes de saturation voire de dégradation de certains sites (Tichnell et al., 1983; Héritier, 2002) nécessitant des réflexions de fond pour tenter de résoudre les impacts liés à l'usage des parcs dans le monde en général, et en Amérique du Nord en particulier (Nelson et al., 1979; Tichnell et al., 1983; Machlis and Tichnell, 1985; Hummel, 1989). La «relation problématique» (Marsh, 1983) entre le tourisme et la conservation constitue un invariant majeur identifié dans la plupart des pays du monde (Nepal, 2000) avec une intensité variable selon la fragilité écologique ou culturelle des sites concernés (Sacareau, 1999). Les conséquences territoriales, prenant en considération les modalités de fonctionnement de l'espace géographique, ont été analysées très précisément 
pour le Canada grâce à l'étude de la vallée de la Bow (Banff-Bow Valley Study, 1996) qui offrait un laboratoire d'analyse particulièrement intéressant de l'effet des activités humaines sur le fonctionnement d'un environnement protégé. Cette étude entre en résonance avec tous les travaux portant sur les transports (Fay, 1999; Clevenger et al., 2002), la présence et la viabilité des communautés locales résidant à l'intérieur ou à proximité d'un espace-parc (Fortin et Gagnon, 1999; Draper, 2000; Héritier, 2006c ; Gagnon et Gagnon, 2006).

À l'instar de la France, où les conflits autour des parcs et les polémiques liées à une gestion dominée par les positions des naturalistes sont particulièrement vifs (Lahaye, 2006 ; Laslaz, 2005), les parcs nationaux nord-américains sont au cœur de revendications d'usages complexes et de fortes tensions entre acteurs. Aux États-Unis comme au Canada, la doctrine de l'usage raisonnable des ressources (doctrine of usefulness) a cédé la place, depuis les années 1960 à une doctrine plus radicale de la protection de la nature, puis de l'environnement et de la biodiversité (Worster, 1998; Duban, 2000; Schmidt di Friedberg, 2004). L'histoire des parcs nationaux nord-américains (Lothian, 1987; Dearden et Rollins, 1993 ; Sellars, 1997) ainsi que l'examen des plans de gestion récents des parcs (Park Service, 1972; Parcs Canada, 2000a, 2000b et 2004; National Park Service, 2004; National Park Service, 2007) montrent clairement que, de 1872 à 2007, les parcs ont vu évoluer les conditions et les législations de la protection: elles ont globalement été caractérisées par le durcissement de la protection de l'environnement, une tendance observable dans l'évolution législative comme dans les missions des parcs. Ainsi la vision de Parcs Canada, c'est-à-dire sa doctrine, consistant «à préserver les milieux qui représentent la diversité [du] territoire [canadien] (Parcs Canada, 1998), a été renforcée par les travaux et les recommandations de la commission sur l'intégrité écologique (Agence Parcs Canada, 2000). Cette situation a radicalisé les positions des deux agences quant à la présence humaine et aux activités dans les parcs, considérées comme des menaces, renforçant de fait les tensions induites pas les objectifs de développement à l’intérieur d'une d’aire protégée.

Dans ce contexte où la protection est clairement dominée par une doctrine écocentriste, l'objectif de cet article consiste ainsi à réfléchir sur les enjeux associés à la mise en place d'une dialectique protection-gestion dans les parcs nationaux nord-américains et ses conséquences en termes de gestion ou d'usages des aires protégées. Fondement du développement national autant que de la protection de l'environnement, les parcs nationaux sont d'abord des terrains de jeu nationaux (1). La variété des activités et leur multiplication vont d'ailleurs progressivement augmenter les tensions entre les utilisateurs (2). Celles-ci révèlent des enjeux majeurs, aujourd'hui encore, et qui nécessitent un recours croissant à la réglementation pour réguler les pratiques dans les parcs (3).

\section{Des terrains de jeu nationaux}

Depuis plus d'un siècle, les parcs sont les lieux d'une intense fréquentation touristique (dominée dans un premier temps par les élites sociales, économiques et culturelles du moment). L'intérêt pour les espaces de grande nature sauvage (wilderness) s'est 
d'autant moins démenti (Nelson et al., 1979; Marsh, 1983) que les parcs ont progressivement été dotés de valeurs (paysagères, éthiques, nationales, etc.) qui les ont amenés à participer du patrimoine national au Canada comme aux États-Unis (Konrad, 1986).

\section{De l'importance de la fréquentation: 100 parcs pour 100 millions de visiteurs}

En 2006, le nombre de parcs nationaux atteint un total de 100 unités (43 au Canada et 57 aux États-Unis). La superficie protégée par les lois sur les parcs nationaux est non négligeable: près de $245000 \mathrm{~km}^{2}$ au Canada en 2006 et plus de $341000 \mathrm{~km}^{2}$ en 2003 aux États-Unis ${ }^{3}$ (tableau 1). La répartition géographique met en évidence deux gradients, l'un septentrional et le second occidental: les parcs canadiens sont d'autant plus vastes qu'ils sont situés aux hautes latitudes (les parcs de Wood Buffalo ([Alberta et Territoires du Nord-Ouest] et Quttinirpaaq [Nunavut] ont pratiquement la même superficie que la Suisse). Aux États-Unis, le gradient est à la fois occidental et septentrional: les parcs sont d'autant plus grands qu'ils sont situés vers l'ouest et vers le nord ${ }^{4}$. Dans les deux cas, une partie de l'explication tient à une caractéristique, celle de la propriété foncière: ils sont en général situés sur des terres appartenant à l'État (au Canada, un parc national doit relever du seul pouvoir fédéral, ce qui conduit à des politiques d'acquisitions foncières très importantes dans les provinces et ce qui contribue à leur donner un caractère politique (Québec), suscitant parfois des débats publics intenses). Aux États-Unis, une partie des parcs ont été créés sur des terres fédérales, même si, dans certains cas, il subsiste quelques îlots privés.

\section{Tableau 1 Les parcs nationaux en Amérique du Nord: données principales}

\begin{tabular}{l|c|l|l|l|l|l|l}
\hline Pays & $\begin{array}{l}\text { Nombre } \\
\text { de parcs }\end{array}$ & $\begin{array}{l}\text { Superficie } \\
\text { totale } \\
\text { des parcs } \\
\text { (arrondi } \\
\text { aux milliers } \\
\text { de km²) }\end{array}$ & $\begin{array}{l}\text { Superficie } \\
\text { moyenne } \\
\text { théorique }\end{array}$ & $\begin{array}{l}\text { Part des } \\
\text { parcs } \\
\text { nationaux } \\
\text { par rapport } \\
\text { à la superficie } \\
\text { totale du pays }\end{array}$ & $\begin{array}{l}\text { Parc le plus } \\
\text { visité } \\
\text { (millions } \\
\text { de visiteurs) }\end{array}$ & $\begin{array}{l}\text { Plus grand parc } \\
\text { national }\left(\mathbf{k m}^{2}\right)\end{array}$ & $\begin{array}{l}\text { Plus petit parc } \\
\text { national }\left(\mathbf{k m}^{2}\right)\end{array}$ \\
\hline Canada & 43 & 245000 & 5697,7 & $2,46 \%$ & $\begin{array}{l}\text { Banff } \\
(3,2)\end{array}$ & $\begin{array}{l}\text { Wood Buffalo, } \\
\text { Alberta et } \\
\text { Territoire du } \\
\text { Nord-Ouest } \\
(44802)\end{array}$ & $\begin{array}{l}\text { Îles du Saint- } \\
\text { Laurent, } \\
\text { Ontario } \\
(8,7)\end{array}$ \\
\hline États-Unis & 57 & 341000 & 5982,5 & $3,64 \%$ & $\begin{array}{l}\text { Great Smoky } \\
\text { Mountain } \\
(20,4)\end{array}$ & $\begin{array}{l}\text { Wrangell-Saint } \\
\text { Elias, Alaska } \\
(53288,6: \text { PN + } \\
\text { National Preserve) }\end{array}$ & $\begin{array}{l}\text { Hot Springs, } \\
\text { Arkansas } \\
(22,46)\end{array}$ \\
\hline
\end{tabular}

Ces parcs sont dotés d'une notoriété nationale - parfois internationale pour certains d'entre eux - considérable: est-il besoin de citer Yellowstone, Yosemite, Grand Canyon? Leurs noms sont familiers partout en Occident, sans doute parce qu'ils sont l'objet d'une intense fréquentation touristique nationale (plus de 87,6 millions de visiteurs dans les parcs états-uniens en $2005^{5}$ ) et parce qu'ils constituent des passages obligés 
de la plupart des opérateurs touristiques internationaux - sans compter les revues grand public - travaillant en Amérique du Nord. Tout aussi remarquables, les parcs canadiens sont moins fréquentés (12,4 millions d'entrées en 2005) (Parcs Canada, 2006) par les visiteurs internationaux européens et assez fortement par les asiatiques, notamment japonais. Toutefois, la notoriété internationale ne constitue pas, à elle seule, un gage de forte fréquentation. Par exemple, aux États-Unis, le parc national Great Smoky Mountain situé à proximité relative d'un gigantesque bassin d'émission touristique avec la megalopolis, accueille 20,4 millions de visiteurs en 2005, alors que la fréquentation de Grand Canyon, de Yellowstone et de Yosemite se situe dans une tranche comprise entre 3,3 et 4,4 millions de visiteurs par an ${ }^{6}$.

Cette fréquentation est également caractérisée par une forte irrégularité à la fois saisonnière et spatiale: saisonnière en raison d'une période de fréquentation principale qui s'étend du 15 juin au 15 septembre en règle générale; des différences persistent toutefois, selon la latitude et la proximité d'une grande agglomération, raisons pour lesquelles certains parcs (ex.: Great Smoky Mountain, La Mauricie) connaissent une fréquentation régulière de fin de semaine plus forte que les autres parcs. Les déséquilibres spatiaux internes sont liés aux conditions d'aménagement des parcs. Ceux-ci étant supposés assurer la protection des écosystèmes qui s’y trouvent, ils sont dotés d'une accessibilité inégale, fondée sur des réseaux routiers déséquilibrés: les sites de visites sont reliés entre eux par des axes de transport de nature et d'importance variables. Cette organisation a pour conséquence de concentrer dans l'espace - et dans le temps - des effectifs élevés de visiteurs.

Dans ce contexte, protéger un espace soumis à une intense fréquentation - fut-elle saisonnière - constitue un véritable tour de force pour des services fédéraux, National Park System (NPS) et Agence Parcs Canada, qui doivent gérer des espaces considérés dans les deux pays comme un patrimoine national, un patrimoine combinant des composantes anthropiques (sites archéologiques, lieux historiques) et des écosystèmes.

\section{Des espaces protégés dévolus au tourisme et à l'appréciation du patrimoine}

Konrad (1986) a bien montré la manière dont un lent processus de construction a conduit à considérer les parcs nationaux en Amérique du Nord comme des éléments clés d'un patrimoine fondé sur des monuments de la nature. Jean Viard écrit à propos des États-Unis qu'ils fondent « [leur] identité dans l'espace, face à une Europe qui possède la durée» (Viard, 1985: 172). Le Canada est assez proche des États-Unis sur ce point précis : les parcs nationaux participent de l'élaboration d'un sentiment national (Konrad, 1986) en tentant d'arrimer les populations locales, régionales et nationales à l'espace protégé. Cette construction d'une relation particulière aux lieux, dont Tuan (1990) parle en proposant la notion de topophilie, légitime et renforce les pratiques de médiation (dont l'interprétation) mises en place par les agences à l'attention des visiteurs (Héritier 2002; Héritier, 2006a et 2006b).

La représentation de la nature (associée aux parcs) et celle de la nation ont elles-mêmes progressivement été élaborées à travers l'art. Dans un premier temps, il fut nécessaire de construire le regard porté sur les paysages "par un travail de déchiffrement des paysages», pour reprendre l'expression de Conan (1985: 181). Cette construction a 
d'abord été réalisée par des peintres paysagistes en Nouvelle-Angleterre. Au Canada, le premier gouverneur général, Lord Dufferin, soutint les peintres paysagistes qui, selon lui, contribuaient à construire les images de la nation naissante (Reid, 1979; Lasserre, 1998). Les photographes participèrent également de ce mouvement. Aux États-Unis des artistes peintres, tels que Thomas Moran, jouèrent un rôle considérable dans la création d'une approche sensible des représentations de la nature dans les parcs (Magoc, 1999: 19). Moran rendit ainsi célèbre les chutes de la rivière Yellowstone, ou le Old Faithful - et au-delà, le parc. Le travail des artistes (Conan, 1985 ; Héritier, 2006b) contribua à la création d'un art national où la recherche métaphysique et la quête nationale se rejoignirent. Le Groupe des Sept, au Canada, constitue l'une des expressions les plus caractéristique de ce processus. Au Canada comme aux États-Unis, les parcs servent à construire une certaine représentation de la nation en condensant une partie de ses valeurs et du rêve national (Héritier, 2002; Schmidt di Friedberg, 2004). Pratique contemporaine, la photographie individuelle remplace le talent du peintre et constitue une forme contemporaine d'appropriation ou de réappropriation des paysages.

Ces parcs participent du patrimoine de la nation pour d'autres raisons : parce qu'ils sont liés aux grandes aventures territoriales (conquête de l'Ouest, colonisation, équipement des axes terrestres, principalement ferroviaires, etc., pour les États-Unis comme pour le Canada) ou parce qu'ils participent de la construction de la représentation nationale du territoire: si le parc national Yellowstone fut créé pratiquement un siècle après l'indépendance, la réserve qui devint le parc national Banff l'est seulement 18 ans après l'Acte de l'Amérique du Nord britannique, qui marque la création du Canada. Dans ce cas précis, il est associé au premier symbole de l'unification du territoire, la voie ferrée et la compagnie Canadien Pacifique.

Enfin, ces espaces bénéficient aujourd'hui de labels nombreux qui les intègrent de facto dans la sphère du patrimoine: plusieurs sont concernés par le programme Man and Biosphere (MAB), par l'inscription sur la liste du Patrimoine de l'Humanité (Unesco), ou encore par l'action d'organisations non gouvernementales (ONG) qui attribuent le label de Parc de la Paix (signalons ici le rôle décisif du Rotary) (Héritier, 2004). Ils traduisent l'intérêt national et supranational porté à ces espaces protégés, présupposant en partie les tensions qu'ils suscitent depuis leur création.

\section{Des tensions autour des usages}

En raison des potentiels que les parcs recèlent, leur histoire a été marquée par des tensions très fortes pour l'utilisation des ressources (minières, forestières, énergétiques, etc.). Ces tensions ne semblent pas s'apaiser et les convoitises se renforcent à mesure de l'augmentation des menaces qui pèsent sur l'accès aux ressources à différentes échelles. Aussi, les administrations tentent de contrôler les usages de manière à maintenir l'adéquation entre les principes de protection et les besoins des sociétés locales. 


\section{Les tensions initiales}

Des tensions sont liées aux conditions de création et d'établissement des parcs nationaux. Jusque dans les années 1960, même si les parcs avaient été créés sur des terres fédérales, un certain nombre d'activités se déroulaient dans les régions concernées: les exploitations forestières (directement opérées par l'État ou en concessions privées), les exploitations minières, l'exploitation hydroélectrique liée à la construction barrages-réservoirs, etc., furent très nombreuses (Bella, 1987; Lothian, 1987 ; Sellars, 1997 ; Louter, 1998 ; Dilsaver et Wycoff, 2005). Dans certains cas, elles contribuèrent à modeler les limites des parcs.

Dans les Rocheuses canadiennes par exemple, les limites des parcs changèrent entre cinq et sept fois pendant la période 1885-1958 (synthèse cartographique dans Héritier, 2002: 318-321). Aux États-Unis, les conditions d'établissement des limites du parc national Olympic dans l'État de Washington furent l'occasion de luttes d'influences considérables entre les différents acteurs régionaux et nationaux. Les ressources forestières, minières et hydrauliques risquaient d'être soustraites aux compagnies d'exploitation, ce qui entraîna de longues oppositions pour l'établissement des limites définitives du parc... particulièrement irrégulières (Lien, 1991; Sellars, 1997). Mais jusque dans les années 1930 au moins, les entrepreneurs conservèrent des droits d'exploitation dans les secteurs aux marges de l'écoumène comme par exemple dans les parcs nationaux Prince Albert (Saskatchewan) ou de La Mauricie (Québec), où l'exploitation forestière fut très importante. Au Saguenay (Québec), les controverses entre l'État fédéral et le gouvernement provincial entre 1976 et 1982 eurent pour effet une construction combinant le parc québécois du Saguenay (aujourd'hui parc national du Québec) au parc marin du Saguenay-Saint-Laurent (dépendant de l'administration fédérale) (Sanguin, 1989). Par ailleurs, comme souvent dans les régions peuplées de longue date, les limites furent longtemps discutées, notamment par les communautés locales dont la vie devait être inégalement affectée par les activités liées au parc (Fortin et Gagnon, 1999).

Cette situation est commune à bien des parcs où les ressources du sol, du sous-sol et de l'hydrosystème étaient - et sont encore parfois - considérées à différentes échelles et à différents niveaux de décision (locaux, régionaux et fédéraux) comme des supports de l'activité économique locale (Fortin et Gagnon, 1999) et nationale... voire comme le support des efforts patriotiques. Certains parcs accueillirent des camps d'entraînement (dont Yosemite, Isle Royale, Death Valley), d'autres furent dotés d'équipements de défense militaire (dont Acadia et Olympic), d'autres encore, dotés de ressources minérales, furent ainsi exploités pendant la Seconde Guerre mondiale: au nom de l'effort de guerre, des mines de sel furent ouvertes dans le parc national Death Valley (Utah), une mine de tungstène dans le parc Yosemite (Californie) (Sellars, 1997: 151-152). La doctrine des usages (doctrine of usefulness) et des prélèvements diversifiés, mais raisonnés dans les espaces protégés (héritée notamment de G. Pinchot, directeur du Forest Service) fut de rigueur jusqu'aux années 1960 aux États-Unis. Au Canada, elle fut très inégale selon les parcs et les périodes, certains parcs voyant interdire les exploitations minières dès le début du XXe siècle (Banff, Jasper), tandis que d'autres (Yoho) poursuivaient l'exploitation forestière après la Seconde Guerre mondiale. Ce durcissement de la législation relatives aux parcs s'affirma dans les années 1960 (Wilderness Act américain et politique des parcs nationaux canadiens en 1964), imposant 
l'approche écologique de la protection et de la gestion des milieux, comme le révèlent les travaux de la Commission sur l'intégrité écologique des parcs nationaux du Canada et la publication du rapport sur l'intégrité (Agence Parcs Canada, 2000).

\section{Les tensions contemporaines}

Les tensions contemporaines entrent en quelque sorte en résonance avec les situations passées dans la mesure où les parcs nationaux constituent - quel que soit leur statut - des réserves d'espaces patrimoniaux, d'écosystèmes et des réserves de ressources. Les exploitations forestières sont toujours la cible privilégiée des défenseurs de l'environnement lorsqu'elles menacent les parcs (exploitation à Wood Buffalo jusque dans les années 1990). Les perturbations viennent aujourd'hui de l'extérieur des parcs, car les environs immédiats des parcs recèlent des ressources considérables qui suscitent la convoitise des sociétés d'exploitation. Ainsi, les observations de terrain effectuées dans les années 1990 montraient que des coupes forestières à blanc étaient opérées aux limites mêmes des parcs nationaux Pacific Rim (ColombieBritannique) et Kootenay; dans les années 2000, aux marges d'Olympic et de North Cascades. Les associations de défense de l'environnement - et les administrateurs des parcs - contestent cette détérioration des espaces hors parcs en utilisant l'idée que les écosystèmes ne s'arrêtent pas aux limites des parcs, et que les effets en retour sont catastrophiques pour les parcs (et notamment pour la grande faune), situation qui met en évidence que les parcs et leurs marges deviennent des enjeux territoriaux et géopolitiques considérables à toutes les échelles.

Les tensions principales sont toutefois liées à l'activité touristique dans les parcs. Cette activité, développée depuis l'origine sous l'impulsion des compagnies ferroviaires, tant aux États-Unis qu'au Canada, a contribué à installer des centres d'accueil (c'està-dire des lieux de superficie restreinte destinés à accueillir les visiteurs), disposant des infrastructures et des fonctions réduites aux activités touristiques principales: hébergement (limité) et commerce de détail. Certaines ont évolué en communautés permanentes et elles sont parfois devenues des stations touristiques, voire des villes. C'est le cas dans l'Ouest canadien où le développement touristique s'est appuyé sur des centres d'accueil qui sont parfois devenus des villes à part entière, comme Banff ou Jasper dans les parcs du même nom. Les problèmes rencontrés sont alors des problèmes urbains (gestion des flux, des pollutions, des eaux, des déchets, etc.) et plus seulement des problématique spécifiques aux parcs nationaux, mais qui s'y ajoutent pourtant (Draper, 2000; Héritier, 2006c). Pour Lowry (1994: 174-180), Banff et Yosemite rencontrent des problèmes similaires, l'un en raison de la présence d'une ville-centre d'accueil qui concentre les visiteurs, l'autre en raison de l'importance du trafic routier dans la vallée de la Yosemite.

Héritage des premières années de mise en valeur, la propriété privée a été définitivement supprimée dans les parcs nationaux canadiens en 1988 (Loi sur les parcs nationaux) ; ce n'est pas le cas aux États-Unis. Près des trois quarts des parcs nationaux américains contiennent des parcelles privées. L'emprise spatiale de ces parcelles peut paraître négligeable puisque certains parcs nationaux ont des terrains privés occupant entre moins de $1 \%$ et $20 \%$ de leur surface. Cette situation est toutefois déterminante dans la gestion de l'espace-parc, notamment dans les parcs nationaux situés en 
Californie, en Ohio, dans les Îles Vierges et en Alaska. Le parc national WrangellSaint Elias (Alaska) et sa réserve comptent près de $16 \%$ de terrains privés (11\% pour le parc national et $16 \%$ pour le parc national et sa réserve, d'après les statistiques du National Park Service de 2003). Étant donné qu'il s'agit du parc national le plus grand des États-Unis (quatre fois Yellowstone sans la réserve et six fois avec celle-ci), ce pourcentage représente toutefois une surface significative. Les problèmes afférents à ces situations sont liés aux conditions d'accès, au déplacement des véhicules et à leur cortège d'altération de l'environnement. Cette situation, présentée comme un paradoxe insoluble entre tourisme et protection, est récurrente dans la plupart des espaces protégés et ce d'autant plus que les parcs sont devenus des produits d'appels du tourisme de nature et des fleurons de l'écotourisme, comme le montrent Gagnon et Gagnon (2006).

Les perturbations externes, enfin, sont de nature différente selon les parcs, leur localisation et la présence d'espaces urbanisés à proximité des parcs. Dans le cas du Cape Cod National Seashore, Claire Leduc (2005) a montré les problèmes induits par la proximité d'un espace exceptionnellement urbanisé (celui de la mégalopolis) et les problèmes de gestion de l'espace protégé. On retrouve rarement une telle concentration urbaine pour la plupart des parcs nationaux. Ils sont toutefois très souvent concernés par les problèmes du développement urbain régional ou local comme dans le cas des villes portes (gateway communities): la ville de Hot Springs regroupe près de 33000 habitants à la limite du parc national du même nom dans l'Arkansas (National Park Service, 2007). À cela s'ajoute les problèmes de la mobilité, induite par les déplacements touristiques, permise par l'amélioration des conditions de locomotion (véhicules individuels) et des conditions de circulation (amélioration du système des transports jusqu'à l'intérieur même des parcs). À titre d'illustration, dans le parc national Everglades (Floride), la modernisation de la route menant à Flamingo situé dans le cœur du parc fut, au début des années 1950, à l'origine de l'explosion touristique (Sellars, 1997: 185-186) ; l'existence actuelle de 82 milles de routes goudronnées (National Park Service, 2000) favorise une fréquentation élevée et une forte pression sur l'écosystème. À cela, ajoutons encore des tensions liées à la présence de propriétés et d'exploitations agricoles (agriculture intensive et élevage extensif) aux limites immédiates des parcs et qui posent des problèmes d'attraction de la faune protégée à l'extérieur : les ongulés cherchent de l’herbe grasse et les prédateurs (ours, loups, couguars) y trouvent des proies faciles, multipliant également les risques de conflits avec les communautés installées à proximité immédiate des parcs (Keiter et Boyce, 1991 : 331 et suiv.).

Ces tensions montrent à quel point les parcs nationaux sont pleinement insérés dans des enjeux liés aux usages dont ils sont le support et qui déterminent en partie la réflexion sur les usages acceptables de cet espace protégé.

\section{Des usages compatibles avec la protection de la nature?}

Depuis l'origine des parcs, ceux-ci ont participé d'une manière ou d'une autre au développement économique national (sans parler des logiques locales et régionales). Entre 1969 et 1988 (1964 pour les États-Unis; 1972, puis 1988 pour le Canada), les activités autorisées dans les parcs nationaux sont davantage orientées vers le tourisme, notamment pour l'hébergement, le déplacement des personnes et des marchandises 
vers les centres d'accueil, les activités de nature (de la simple contemplation des paysages aux pratiques sportives diverses: escalade, cascades de glace, sports d'eau vive, etc.). L'accueil et l'hébergement constituent des enjeux majeurs dans la mesure où, au Canada comme aux États-Unis, l'une des missions des parcs nationaux concerne la satisfaction des visiteurs.

Les objectifs des deux agences sont en la matière assez voisins: le National Park Service (États-Unis) est chargé, depuis 1916, de "conserver le paysage, ainsi que les éléments naturels, historiques et la faune sauvage qui s'y trouvent, et en faire profiter le public d'une manière et par des moyens qui les laissent intacts pour le profit des générations futures» (US code, art. 16, section 1, cité dans Leduc, 2005 : 62) ; le service canadien des parcs, au regard de la Loi sur les parcs nationaux de 1988 dédie les parcs nationaux au "peuple canadien afin que celui-ci puisse les utiliser pour son plaisir et l'enrichissement de ses connaissances (...); ils doivent être entretenus et utilisés de façon à rester intacts pour les générations futures» (Parcs Canada, 2000a: 4). Il existe toutefois des usages, a priori inattendus au regard du souci de conserver intact les lieux. Cet objectif est hérité de l'histoire des parcs et de la place qu'ils ont occupée dans le développement économique régional et national. Les activités de transport, nécessaires au déplacement des visiteurs, sont particulièrement touchées: routes traversières, voies ferrées transcontinentales, routes permettant l'accès aux sites, domaines skiables équipés des remontées mécaniques, stations thermales (Mammoth Hot Springs, Banff), grands hôtels construits par les compagnies ferroviaires, etc.

Les usages sont aujourd'hui dominés par les activités récréatives et touristiques de visiteurs, par définition non résidants. La fréquentation a pour caractéristique d'être temporaire et de limiter le volume des aménagements. Dans quelques rares cas, les populations locales résident dans les parcs. Héritier (2006c) a exposé les conditions dans lesquelles quelques collectivités et quelques centres d'accueil s'étaient maintenus dans certains parcs canadiens. Dans certains cas, les communautés résidentes (notamment autochtones) disposent de droits spécifiques - cette question rejoint celle des revendications autochtones relatives aux droits traditionnels ou ancestraux. Elles font valoir d'anciens droits d'usages (droits cynégétiques notamment) sur des terres dont elles revendiquent la propriété ou au moins l'usufruit. Cet aspect a été en partie anticipé pour les parcs situés aux marges de l'écoumène, dans les régions septentrionales: dans les parcs nationaux situés dans les territoires du Nunavut, du Nord-Ouest, du Yukon, les populations préexistantes conservent un certain nombre de droits traditionnels. Il en va de même en Alaska où l'Alaska National Interest Lands Conservation Act $^{7}$ (ANILCA) fut voté le 2 décembre 1980, quelques semaines avant l'investiture du président républicain Ronald Reagan. Cette loi, résultant d'un compromis entre les associations environnementalistes et le gouvernement démocrate du président Jimmy Carter, autorisa des usages généralement interdits, tels que la prospection pétrolière (Lowry, 1994: 71). Elle autorisa également les activités de subsistances considérées comme des « usages traditionnels et coutumiers des résidents ruraux en Alaska » : c'est-à-dire un droit de prélèvement pour les ressources destinées à la survie: nourriture, habitat, combustible, vêtements, objets, ou transport, et pour la fabrication et la vente dans le cadre de l'artisanat (chapitre VIII, paragraphe 803 de l'Alaska National Interest Lands Conservation Act). 
Figure 2 Enjeux territoriaux associés aux parcs nationaux WrangellSaint Elias et Kluane

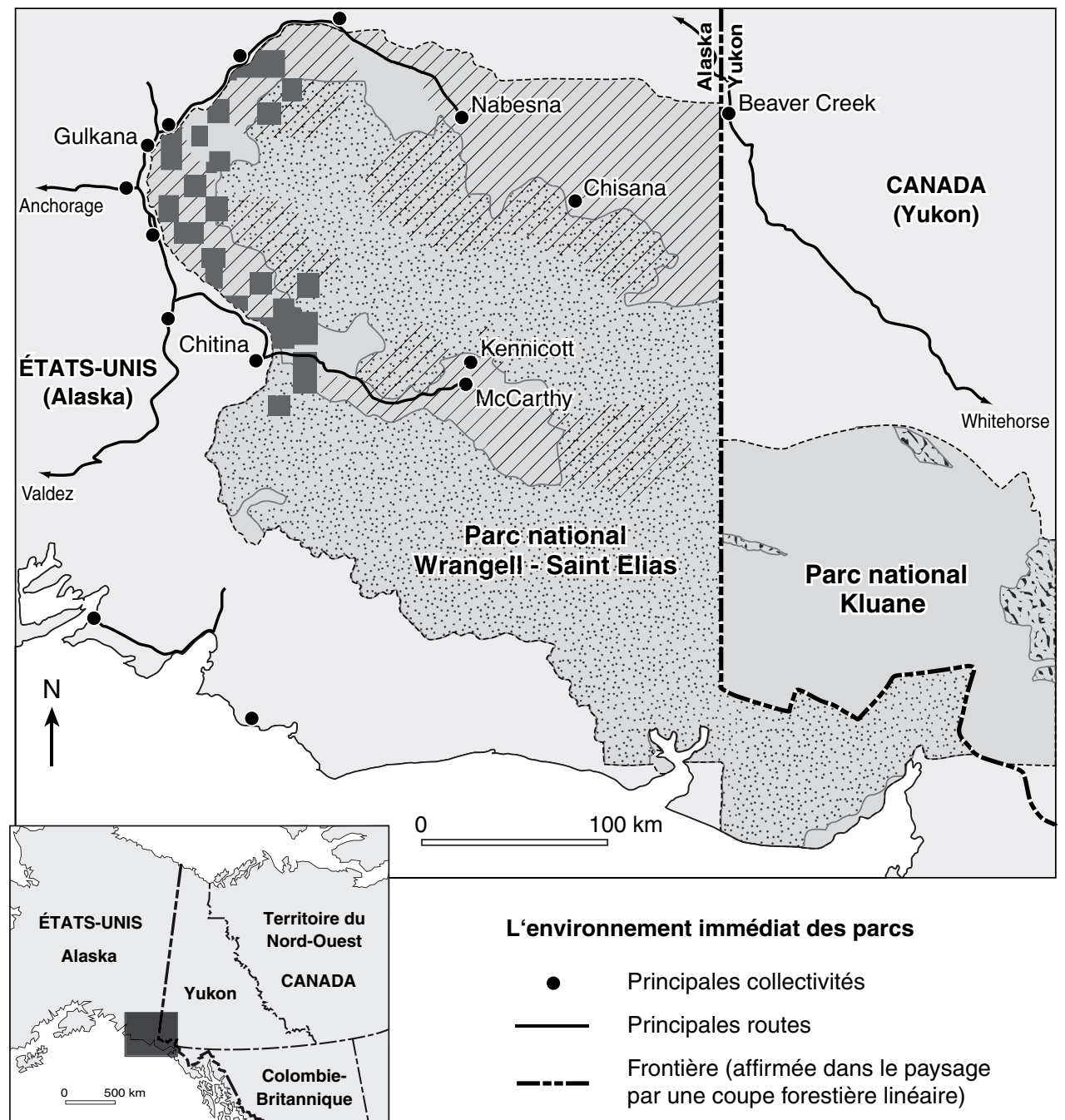

C. Moumaneix et S. Héritier, 2006,

d'après : Carte de Wrangell-Saint Elias (NPS) et Parcs Canada, 2004.

\section{Limites et divisions des parcs}

\section{Limites de Parc National}

Propriétés privées à l'intérieur du parc Wrangell-Saint Elias

Aire de nature sauvage (Wilderness Area)

Zone de préservation spéciale (Zone I dans la nomenclature de zonage en vigueur à Parcs Canada)

National Preserve 
Les tensions demeurent toutefois entre les populations locales et l'agence fédérale (Bleakley, 2002). Les contours du parc Wrangell-Saint Elias traduisent clairement les stratégies territoriales d'évitement vis-à-vis des communautés locales préexistant aux parcs. À titre d'illustration, Moumaneix (2006a et b) observe que le dessin des limites du parc contourne soigneusement un chapelet de communautés rurales locales (figure 2). Mais quatre communautés n'ont pu être écartées, en raison de leur situation centrale par rapport au parc. Les autorités ont ainsi effectué un zonage à l'intérieur du parc - spécifique à l'Alaska - pour exclure les communautés de la surface libellée National Park et de la zone de Wilderness. Cette situation demeure pourtant exceptionnelle et la politique générale des deux agences, fondée sur une conception de l'environnement très écocentriste, est plutôt exclusive, comme en témoignèrent les rudes expropriations au moment de la création des parcs nationaux Forillon et Kouchibouguac (Fortin et Gagnon, 1999). Par ailleurs, ces expériences montrent que la doctrine écocentriste ne considère que rarement les sociétés humaines comme étant partie intégrante de l'environnement, ce que n'excluaient pourtant pas les pères de l'écologie (Worster, 1999).

Les tensions exposées dans les paragraphes précédents révèlent l'ampleur des enjeux qui se jouent autour et au sujet de ces espaces protégés. Ils révèlent d'autres enjeux qui méritent attention.

\section{Enjeux contemporains autour du renforcement des législations nationales}

Les tensions concernant les parcs nationaux demeurent vives. Les projets d'ouverture des réserves sauvages dans le nord de l'Alaska à l'exploitation pétrolière (en 2005) ont rappelé la fragilité politique des aires protégées quand bien même il semblait exister un consensus social devant assurer leur pérennité. Perspective d'autant plus traumatisante que l'opinion publique états-unienne pro environnementaliste se souvient du naufrage de l'Exxon Valdez en 1989, souillant, entre autres, les côtes du parc national Kenai Fjord.

Dans une certaine mesure, il paraît alors légitime d'insister sur deux éléments qui occupent une place centrale dans la réflexion sur les parcs nationaux. D’une part, ils apparaissent comme des outils essentiels de la maîtrise territoriale. D'autre part, en dehors des tensions liées à l'exploitation des ressources, les enjeux actuels portent sur les activités touristiques et sur les tentatives de gestion concertée entre les deux pays, qui dépassent l'échelle et le seul niveau de compétence des parcs.

\section{Parcs nationaux et maîtrise territoriale}

Il ne fait pas de doute que les parcs nationaux sont des espaces appropriés par des groupes sociaux souvent concurrents. La question de la maîtrise territoriale se pose alors de manière cruciale. Qui contrôle les parcs? Et à quelle échelle cette maîtrise s'opère-t-elle? D'une manière générale, la première question peut trouver une réponse générale simple: les États fédéraux, par l'intermédiaire de services ou d'agences sous la tutelle de l'autorité fédérale. Celles-ci font valoir leur autorité et fixent les cadres juridiques par la promulgation de lois ayant trait directement ou ayant des inciden- 
ces indirectes sur les parcs nationaux: la période 1960-1980 a été particulièrement féconde sur ce point aux États-Unis, et l'on ne compte pas moins de dix lois sur la question (dont le Wilderness Act en 1964, le National Environmental Policy Act en 1969, le Clean Air Act en 1977, le Clean Water Act en 1977 ou ANILCA en 1980, etc.). Au Canada, la période la plus active se situe entre 1984 et 2006: la loi sur les parcs nationaux (votée en 1988 par le Parlement) actualise une loi qui datait de 1930 et qui s'avérait peu adapté à la situation et aux besoins contemporains de protection. Le Plan vert du Canada pour l'environnement en 1990 fixait des objectifs nationaux en termes de protection de l'environnement, arsenal législatif complété par la Loi canadienne sur la protection de l'environnement (en 1999) et les différents amendements à la Loi sur les parcs nationaux en 1998, en 2000 (Dearden et Dempsey, 2004: 231) ou encore en 2004.

Ces réglementations ont pour objectif de permettre une maîtrise efficace des activités à l'intérieur des parcs. Dans les deux pays, les parcs établissent - dans les limites de la loi fédérale - des plans directeurs ou plans de gestion qui définissent les objectifs de la protection et de l'utilisation des parcs ainsi que leurs missions. Ils fixent des objectifs en matière de diminution de l'impact des activités humaines sur les écosystèmes protégés: ainsi Parcs Canada emploie la notion d'intégrité écologique pour désigner «l'état d'un écosystème jugé caractéristique de la région naturelle dont il fait partie, plus précisément par la composition et l'abondance des espèces indigènes et des communautés biologiques ainsi que par le rythme des changements et le maintien des processus écologiques » (Agence Parcs Canada, 2000: 2). Cette notion en vigueur à Parcs Canada reflète l'écocentrisme de la doctrine fondant l'action de protection de l'agence. À partir de ce principe, les parcs établissent des systèmes de zonage rigoureux de l'espace (Dearden et Dempsey, 2004: 228), établissant des niveaux acceptables d'utilisation de l'espace et «Parcs Canada fut encouragé à réaliser un zonage qui était plutôt fondé sur l'utilisation que l'on désirait et moins sur celle qui était en vigueur » (Hodgins et al., 2000: 286; pour des exemples, se référer à Parcs Canada, 2000a et 2000b) ${ }^{8}$. Les deux systèmes proposent un zonage de l'espace avec des évolutions inégales. Ainsi, le parc national Glacier n'a pratiquement pas modifié son zonage depuis 1977. Celui du Mont Rainier a été établi en 1972 et a peu évolué (Park Service, 1972). À l'inverse, le parc Acadia (Maine) présenta en 2004 un plan directeur qui révisant l'ancien zonage dans le sens d'un renforcement significatif de la protection des ressources naturelles et culturelles (National Park Service, 2004: 25). Ailleurs, comme ce fut le cas pour le parc North Cascade (Louter, 1998; Perla, à paraître; Moumaneix, à paraître), les limites du parc furent établies en fonction de certains équipements préexistants (barrages, pistes transformées en routes après la création du parc), ce qui conduisit à la création d'un parc national composé de deux unités, distinctes spatialement, séparées par un «espace national de loisirs» caractérisé par le grand réservoir du barrage du lac Ross situé dans la vallée de la rivière Skagit (National Recreation Area) et bordé au sud par le Lake Chelan National Recreation area.

Le renforcement des mesures de protection a pour conséquence de produire des logiques de reflux d'aménagements: les bâtiments liés aux exploitations anciennes sont détruites ou déplacées lorsqu'ils ne sont pas patrimonialisés. Parfois, certains (gardiens des parcs, acteurs locaux) regrettent que ces pratiques se réduisent à la suppression des aménagements existants (routes, pistes forestières, barrages), tandis que d'autres (associations environnementalistes notamment) regrettent la lenteur 
à effectuer ces suppressions. Cette dernière remarque montre toute la difficulté à laquelle se heurtent les administrations des parcs, en permanence au cœur d'enjeux qui imposent des arbitrages aussi complexes que délicats, sous-tendus ou légitimés par des arguments financiers.

\section{Les usages touristiques de l'espace}

Deux éléments émergent au regard de la fréquentation touristique: le nombre de visiteurs et leurs modes de déplacements (et accessoirement le transport des marchandises utiles aux activités de service). L’augmentation de la fréquentation touristique et, dans certains cas, de la circulation traversière est devenue l'enjeu majeur des années 1990 dans les parcs nationaux nord-américains, alors même que les réglementations en faveur de la protection se voulaient plus rigoureuses.

Rappelons rapidement le rôle essentiel des transports dans le développement et parfois dans la création des parcs de l'ouest et du nord du continent. Des voies ferrées (pour le transport transcontinental de marchandises) sont encore en service à l'intérieur de certains parcs (Banff, Jasper). Ailleurs, elles longent les parcs ou font partie de l'écosystème immédiat (les parcs Mont Revelstoke au Canada, Glacier ou Yellowstone aux États-Unis). Dans le même temps, de grandes routes transcanadiennes (aux dimensions autoroutières, par leur taille et les flux enregistrés) traversent les mêmes parcs nationaux (aux précédents cités, ajoutons les parcs nationaux Glacier et Mont Revelstoke au Canada).

À cette situation s'ajoute partout la même question: y a-t-il trop de véhicules et de visiteurs? Dans une intervention concernant les problèmes induits par la fréquentation dans les parcs nationaux états-uniens, Bruce Babbit, ministre de l'intérieur américain déclarait en 1997: "Some people say there are too many people coming to our national parks. I reject that. I believe there can never be too many people, too many Americans, coming to enjoy their parks. I do believe, though, there are too many cars ${ }^{9}{ }$. La forte présence des touristes - donc des véhicules - a conduit Parcs Canada à développer une réflexion sur le sujet depuis le début des années 1990:

Le transport engendre certains des conflits les plus graves sur le plan de l'utilisation du territoire dans la région des parcs des montagnes et dans toute la région entre Yellowstone et le Yukon. La circulation sur la route transcanadienne $n^{\circ} 1$ a augmenté de $40 \%$ depuis 10 ans. On ne fait qu'aborder les problèmes de transport ayant une incidence sur la faune avec des données exactes. Ils ne seront pas réglés après cinq ans de recherche ${ }^{10}$.

La circulation et l'utilisation des routes sont très inégales. Les deux routes transcanadiennes (ATC 1 et ATC 16) sont les plus fréquentées: les études montrent que la ATC 1 (porte d'entrée est du parc national Banff) voyait passer 14518 véhicules par jour en moyenne (dans les deux sens), avec des pics à 30000 véhicules par jour pendant l'été (Parks Canada, 1997; Clevenger et al., 2002: 1-2). À Jasper, la ATC 16 enregistre pour sa part 3360 véhicules-jour (données fournies par Parcs Canada, Parc National de Jasper). Dans les deux cas, les chiffres étaient nettement plus élevés que ceux de la US-Highway 2 qui passe dans le parc Glacier (Montana) où l'on enregistrait 1600 véhicules-jour (Clevenger et al., 1998: 1-2). 
Les problèmes nés de la fréquentation touristique croissante et du nombre de véhicules sont communs à la plupart des parcs nationaux: les parcs nationaux états-uniens Yosemite (Californie) et Glacier (Montana) créent les systèmes de navettes (Shiftan et al., 2006) - parfois privées comme à Wrangell-Saint Elias (Alaska) ou North Cascades (Washington) - depuis quelques années (Fay, 1999). Le parc national Bryce Canyon propose également « a mass transit system to minimize vehicle congestion and the resulting impacts that jeopardize the very things we all treasure about national

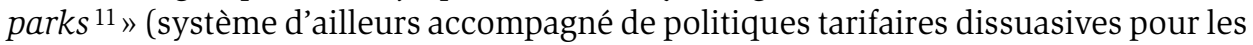
conducteurs de véhicules individuels). Le plan directeur du parc Acadia propose depuis 2004 de mettre en place un programme-test de navettes et d'étendre l'incitation à la pratique de la bicyclette à l'intérieur du parc. Cette question semble d'ailleurs assez commune puisqu'en France, par exemple, la gestion des trafics a été envisagée de manière très diverses selon les sites : recours à des navettes, aires de concentration (ou de stockage) des véhicules (Bernier, 2003) ou paiement du stationnement à proximité des sites les plus visités (Laslaz, 2005).

L'observateur constate que les missions des parcs sont directement affectées, pour ne pas dire menacées, par le succès dont ils sont l'objet. Recherchés, convoités, rêvés, patrimonialisés, ils sont gérés à des échelles qui sont parfois trop réduites - au regard de leurs missions. Cela suggère des arbitrages entre les objectifs administratifs et les intérêts financiers qui doivent concilier le développement humain et la préservation des écosystèmes. La création des corridors écologiques de grande dimension, qui nécessitent d'envisager des coopérations transfrontalières, constitue une tentative de réponse à la fragmentation des écosystèmes.

\section{Une délicate gestion par delà les frontières}

Des tentatives d'actions coordonnées existent depuis 1932, date à laquelle le Rotary (du Montana et d'Alberta) proposa d'établir un parc international de la Paix par la réunion des parcs nationaux Lacs Waterton (Canada) et Glacier (États-Unis) (Héritier, 2004 : 55-58). Jusqu'au début des années 1970, les deux parcs n'avaient pas vraiment développé d'actions spécifiques en faveur de la promotion du parc international de la Paix. Depuis cette période, de réels efforts ont été entrepris, notamment en matière d'échange de personnels d'interprétation entre les deux parcs, de travaux et d'actions coordonnés pour certains événements, de partage des compétences en termes de recherche scientifique, d'éducation des visiteurs ${ }^{12}$ et d'interprétation (exemple, l'International Hike de Waterton à Goat Haunt, menée par un ranger et un warden), de mise en place de réglementations identiques en matière de pêche sur le lac Waterton supérieur, et par le port d'un insigne identique rappelant l'appartenance au parc international (Lieff et Lusk, 1990: 46). Enfin, l'Association du parc international de la Paix (financée par les clubs Rotary d'Alberta et du Montana) poursuit les actions engagées depuis 1932.

Les initiatives communes les plus récentes sont intégrées dans des actions de concertation régionale (parcs, organismes forestiers, nation autochtone Blood, etc.) qui s'appliquent à des aspects de gestion intéressant les deux parcs nationaux, mais aussi leurs espaces périphériques, afin de réduire les menaces extérieures concernant les espèces protégées (Prato, 2003: 47). Ces actions concernent la gestion des incendies de forêts, la lutte contre les plantes non indigènes, l'écotourisme et la gestion des loi- 
sirs dans l'arrière-pays des parcs (Parcs Canada, 2000b). Le statut particulier du parc international favorise les rapprochements entre les responsables canadiens et étatsuniens et la coopération existe également dans les domaines tels que les opérations de recherche et de secours en montagne, d'information sur la sécurité dans l'avant et l'arrière-pays, les conditions météorologiques ou la fermeture des sentiers (Lieff et Lusk, 1990; Timothy, 2002). Au-delà, ils sont intégrés dans un vaste corridor écologique (le Y2Y: Yellowstone to Yukon) s'étendant du Wyoming (Yellowstone, Grand Teton) jusqu'au Yukon et en Alaska, mais il semble que la question de la coopération ne soit pas encore suffisamment avancée pour proposer des résultats significatifs, comme le soulignent des études récentes (Danby et al., 2003; Moumaneix, 2006a et b), dont certaines mettent en évidence les différences en termes juridiques : par exemple, Vásárhelyi et Thomas (2006) montrent que si le Canada s’appuie sur l'intégrité écologique, les États-Unis n'utilisent pas cette notion de manière explicite. Les ententes de principe entre les deux pays ne prévoient pas vraiment de clauses spécifiques pour connecter les aires protégées du corridor écologique. Selon ces auteurs, il manque un véritable traité bilatéral qui permettrait l'élaboration des législations et de politiques de protections conjointes ainsi qu'une véritable stratégie commune.

Dans les faits, la coopération apparaît encore assez limitée. Elle concerne le partage d'informations (migration des caribous entre les parcs Wrangell-Saint Elias et Kluane dans le cadre d'un protocole d'entente signé en 1998 (Parks Canada, 2004: 78) ; la collaboration dans certains domaines tels que la lutte contre les incendies réunissant la Colombie-Britannique et les unités du NPS, du Forest Service et du Bureau of Land Management du nord-ouest des États-Unis à la suite de la signature du Northwest Border Arrangement for Fire Protection le 16 juin 2004; la coordination de projets communs (réintroduction de grizzlis dans le parc North Cascades), des programmes d'interprétation (International Point, Hozomeen, sur la frontière entre North Cascades et le parc provincial Skagit Valley) (Moumaneix, à paraître), mais aucune gestion intégrée de l'écosystème dans son ensemble. Il est possible d'expliquer cette situation par la réactivité lente de chacun des deux systèmes fédéraux, par les déséquilibres entre leurs budgets, par la faible compatibilité des modes de zonage de l'espace, par les conditions de terrain qui rendent difficile une réelle coopération (Alaska), et par la priorité accordée aux questions de gestion interne de la fréquentation touristiques plutôt qu'aux approches concertées.

\section{Conclusion: des espaces entre protection et convoitises}

Les parcs nationaux nord-américains recensent près de 100 millions de visiteurs par an. Ils participent ainsi d'un vaste système touristique - organisé à différentes échelles - qui s'appuie sur la valorisation de la nature, des phénomènes géologiques et géomorphologiques, mais également sur la médiation des rapports entre les sociétés passées et cette nature (à travers des pratiques d'interprétation). Territoires convoités par des activités concurrentes, les parcs sont, depuis l'origine, un sujet d'enjeux particulièrement intenses entre diverses composantes de la société qui découlent de différentes conceptions de l'environnement et de sa gestion et qui, chacune, revendique des usages et des modes de gestion spécifiques. Ces tensions contribuent à produire, à imposer ou à légitimer, selon les cas, des réglementations destinées à réguler les pratiques et les usages de l'espace dans les parcs (stratégies spatiales, stratégies de gestion ou de contrôle des flux, etc.). 
On observe que ces espaces sont toujours inscrits dans une dialectique ou la protection et la gestion se répondent et se légitiment l'une l'autre. Les plans de gestion et les nouvelles pratiques de gestion des visiteurs (surtout) mettent en évidence des dynamiques d'organisation des flux, des activités où l'on perçoit une volonté de prise de contrôle croissante de la fréquentation qui laisse parfois perplexe quant aux objectifs : souhaite-t-on effectivement protéger ces espaces? Ou tente-t-on de proposer des modes de gestion qui offrent de nouveaux débouchés économiques aux opérateurs privés exerçant leur activité à l'intérieur des parcs? La question du transport collectif, justifié par les arguments écologiques, contribue également à constituer des clientèles captives pour les compagnies chargées de desservir certains sites. Elle illustre assez clairement les jeux de forces qui s'opèrent entre les tenants du développement économique, légitimé par la connotation positive de l'écotourisme, et les agences fédérales. Ces dernières disposent de mandats les conduisant à des pratiques de gestion qui peuvent sembler schizophrènes et qui leur attirent, depuis plusieurs décennies, des critiques de toutes parts. Soit qu'elles sont accusées d'empêcher le développement économique par les uns, soit qu'elles sont suspectées par les autres de brader la protection de la nature et la biodiversité aux forces économiques. À la décharge des agences administrant ces parcs, il faut reconnaître que leur mission demeure extrêmement délicate dans des pays où les parcs constituent des icônes nationales et où ils représentent, pour reprendre l'expression de l'écrivain états-uniens Wallace Stegner, a part of the geography of hope.

\section{Remerciements}

Les auteurs souhaitent remercier les quatre évaluateurs anonymes pour leurs commentaires sur la première version de ce manuscrit.

\section{Notes}

1 Ce texte rassemble une partie des résultats de recherches effectuées à l'occasion de plusieurs missions de recherche dans les parcs nationaux canadiens et états-uniens entre 1996 et 2006. Il fait suite à une conférence préparée par les deux auteurs et présentée par Stéphane Héritier à l'occasion du Festival international de géographie de Saint-Dié (28 septembre $-1^{\text {er }}$ octobre 2006) sur les parcs nationaux nord-américains.

2 Dans les deux pays, les théories de la protection (qu'il s'agisse des idées de conservation ou de préservation) vont avoir des incidences sur la manière d'utiliser les ressources naturelles (notamment forestières) au cœur même des parcs nationaux. Ces théories participent des préoccupations des services forestiers - rappelons le rôle de Gifford Pinchot aux États-Unis et de Clifford Sifton au Canada - qui souhaitent gérer les ressources forestières avec raison. Cet usage raisonnable des ressources, correspondant à la conception nord-américaine de la conservation, a été inspiré à la fois par les réflexions de Marsh et par la valeur spirituelle attribuée aux espaces de nature sauvage par le transcendantalisme, conception largement véhiculée par des personnalités telles que John Muir, fondateur du Sierra Club.

3 Ce chiffre correspond aux parcs nationaux, National Preserve, National Wilderness Area, National Lakeshore, etc. et qui comprennent près de $5 \%$ de terres privées. Source: d'après http://www2.nature.nps.gov/stats/acrebypark03cy.pdf

4 Le parc national des Everglades, Floride, constitue une exception à l'est mais sa taille s'explique par la volonté de protéger un vaste écosystème de marais littoral. 
5 Annual visitation report. Source: www2.nature.nps.gov/NPstats (septembre 2006).

6 Annual visitation report. Source: www2.nature.nps.gov/NPstats (septembre 2006).

7 Source: http://www.r7.fws.gov/asm/anilca/intro.html

8 Les parcs canadiens présentent un zonage en cinq zones (I - Préservation spéciale, II - Milieu sauvage, III - Milieu naturel, IV - Loisirs de plein air, V - Services du parc), tandis qu'aux États-Unis, trois zones (ensuite subdivisées, servent au zonage des parcs): Wilderness zones, Diverse Visitor Experience zones, et Developed zones. Dans le cas des parcs canadiens, les principes ont été étudiés et développés par Sanguin et Gill (1991) pour Jasper, Richez (1991) pour Banff, Marchand (2001) pour les parcs littoraux et Héritier (2002) pour les parcs des montagnes de l'Ouest.

9 Bruce Babbitt, Ministre de l'intérieur, cité dans William Booth, Floods Brought Yosemite the Break of the Century, Washington Post, 6 mars 1997, cité dans Quin, 1997, pp. 57-58.

10 Site Internet de Parcs Canada: http://www.pc.gc.ca/pn-np/ab/banff/docs/routes/chap1/ sec5/routes1f_f.asp (le 23/08/06).

11 Source: http://www.nps.gov/archive/brca (23/09/06).

12 L'éducation des visiteurs constitue l'un des points fondamentaux de l'action de médiation des parcs nationaux nord-américains, tant pour les parcs terrestres que pour les parcs littoraux et marins (Marchand, 2001: 111). 


\section{Bibliographie}

Agence Parcs Canada (2000) Intacts pour les générations futures? Protection de l'intégrité écologique par les parcs nationaux du Canada. Vol. I Le temps d'agir. Rapport de la Commission sur l'intégrité écologique des parcs nationaux du Canada, Ottawa.

ARNOULD, Paul et GLON, Éric (dir.) (2005) La nature a-t-elle encore une place dans les milieux géographiques? Paris, Publications de la Sorbonne.

Banff-Bow Valley Study (1996) Banff-Bow Valley: At the Crossroads. Technical Report of the Banff-Bow Valley Task Force (Robert Page, Suzanne Bayley, J. Douglas Cook, Jeffrey E. Green, and J. R. Brent Ritchie). Prepared for the honourable Sheila Copps, Minister of Canadian Heritage, Ottawa.

BELLA, Leslie (1987) Parks for profit. Montréal, Harvest House.

BERNIER, Xavier (2003) Transports, parcs nationaux et parcs naturels régionaux dans les Alpes françaises: les interactions entre les objectifs de l'accessibilité, de la protection et de l'aménagement. Revue de géographie alpine, vol.91, n² 2 , pp. 27-39.

BLEAKLEY, Geoffrey T. (2002) Contested Ground. An Administration History of Wrangell-St.Elias National Park and Preserve, Alaska, 1978-2001. National Park Service, Alaska System Support Office, Anchorage.

BUTLER, Richard W. et BOYD, Stephen W. (dir.) (2002) [2000] Tourism and National Parks, Issues and implications. Chichester, John Wiley and Sons, Ltd.

CADORET, Alain (dir.) (1985) Protection de la nature, histoire et idéologie: De la nature à l'environnement. Paris, L'Harmattan.

CLEVENGER, Anthony P. et al. (2002) Roads and Wildlife in the Canadian Rocky Mountain Parks - Movements, Mortality and Mitigation, November 1996 - March 2002. September 2002. Canmore, Alberta. Report Submitted to Parks Canada, Banff, Alberta.
CONAN, Michel (1985) Découverte et invention de Yellowstone. Esquisse de l'histoire de la création d'une culture visuelle aux États-Unis au XIXe siècle. Dans Alain Cadoret (dir.) Protection de la nature, histoire et idéologie: de la nature à l'environnement, Paris, L'Harmattan, pp. 157-187.

DANBY, Ryan K. et al. (2003) Science and the St.Elias: An Evolving Framework for Sustainability in North America's Highest Mountains. The Geographical Journal, vol. 169, nº 3, pp. 191-204.

DEARDEN, Philip et DEMPSEY, Jessica (2004) Protected areas in Canada: decade of change. The Canadian Geographer, vol. 48, no 2 , pp. 225-239.

DEARDEN, Philip et ROLLINS, Rick (dir.) (1993) Parks and Protected Areas in Canada, Planning and Management. Toronto, Oxford University Press.

DILSAVER, Lary M. et WYCOFF, William (2005) The Political Geography of National Parks. Pacific Historical Review, vol.74, no 2, pp. 237-266.

DRAPER, Dianne (2000) Towards Sustainable Mountain Communities: Balancing Tourism Development and Environmental Protection in Band and Banff National Park. Ambio (Royal Swedish Academy of Sciences), vol. 29, nº 7, pp. 408-415.

DUBAN, François (2000) L'écologisme aux États-Unis : histoire et aspects contemporains de l'environnementalisme américain. Paris, L'Harmattan.

DUBOIS, Jean-Jacques, HOTYAT, Micheline et COURTOIS, Nicolas (2005) Les réserves forestières en France. Dans Paul Arnould et Éric Glon (dir.) La nature a-t-elle encore une place dans les milieux géographiques? Paris, Publications de la Sorbonne, pp. 45-67.

FAY, Bill (1999) Yosemite Shuttle Bus System. Dans National Parks: Transportation Alternatives and Advanced Technology for the $21^{\text {st }}$ Century. Conference Proceedings, Big Sky, Montana. 
FORTIN, Marie-José et GAGNON, Christiane (1999) An Assessment of Social Impacts of National Parks on Communities in Quebec. Environmental Conservation, vol.26, no3, pp. 200-211.

GAGNON, Christiane et GAGNON, Serge (dir.) (2006) L'écotourisme entre l'arbre et l'écorce. De la conservation au développement viable des territoires. Québec, Presses de l'Université du Québec.

GLAZEBROOK, George Parkin de Twenebroker (1964) A History of Transportation in Canada, vol. II. Toronto, McClelland and Stewart.

HÉRITIER, Stéphane (2002) Environnement et patrimoine, tourisme et aménagement dans les parcs nationaux des montagnes de l'Ouest canadien (Banff, Jasper, Yoho, Kootenay, Revelstoke, Glacier, Lacs Waterton). Chambéry, Université de Savoie, thèse de doctorat non publiée.

HÉRITIER, Stéphane (2004) Here, at last, is Peace - Le Parc International de la Paix Waterton-Glacier et la gestion transfrontalière des espaces protégés (Alberta, Canada - Montana, États-Unis). Études canadiennes, no 57 , pp. 51-71.

HÉRITIER, Stéphane (2006a) Phénomènes karstiques et tourisme dans les parcs nationaux de l'Ouest canadien: la mise en valeur progressive d'un patrimoine naturel. Karstologia, nº 47 , pp. 33-48.

HÉRITIER, Stéphane (2006b) La nature et les pratiques de la nature dans les montagnes canadiennes: le cas des parcs nationaux des montagnes de l'Ouest (Alberta et Colombie-Britannique). Annales de Géographie, no649, pp. 270-291.

HÉRITIER, Stéphane (2006c) Espaces urbanisés et parcs nationaux: le défi de la gestion des espaces urbanisés dans les parcs nationaux de l'Ouest du Canada. Norois, no 199, pp. 61-76.

HODGINS, Douglas W. et al. (2000) From Confrontation to Conservation: The Banff National Park Experience. Dans Stephen F. McCool et al. Wilderness Science in a time of change conference, vol.2: Wilderness within the context of larger systems, 1999 May 23-27, Missoula, MT. pp. 281-289.
HUMMEL, Monte (1989) Endangered Spaces: The Future for Canada's Wilderness. Toronto, Key Porter Books.

KEITER Robert B. et BOYCE Mark S. (dir.) (1991) The Greater Yellowstone Ecosystem: Redefining America's Wilderness Heritage. New Heaven, Yale University Press.

KONRAD, Victor (1986) Focus: Nationalism in the Landscape of Canada and the United States. The Canadian Geographer, vol.30, n², pp. 167-180.

LAHAYE, Nathalie (2006) Lorsque conservation et tourisme se confrontent. Le cas du Parc national des Pyrénées et ses conflits d'usages. Dans Christiane Gagnon et Serge Gagnon (dir.) L'écotourisme entre l'arbre et l'écorce. De la conservation au dévelopement viable des territoires, Québec, Presses de l'Université du Québec, pp. 171-203.

LASLAZ, Lionel (2005) Les zones centrales des Parcs Nationaux alpins français (Vanoise, Ecrins, Mercantour), des conflits au consensus social? Contribution critique à l'analyse des processus territoriaux d'admission des espaces protégés et des rapports entre sociétés et politiques d'aménagement en milieux montagnards. Chambéry, Université de Savoie, thèse de doctorat non publiée.

LASSERRE, Frédéric (1998) Le Canada d'un mythe à l'autre. Territoire et images du territoire. Montréal, Hurtubise.

LEDUC, Claire (2005) Entre devoir de préserver et droit d'user, compromis territorial sur la côte mégalopolitaine. L'information Géographique, nº69, pp. 50-66.

LIEFF, Bernard et LUSK, Gil (1990) Transfrontier Cooperation between Canada and USA: Waterton-Glacier International Peace Park. Dans Jim Thorsell (dir.) Parks on the Borderline: Experience in Transfrontier conservation, IUCN, Grand, Switzerland.

LIEN, Carsten (1991) Olympic Battleground. The Power Politics of Timber Preservation. San Francisco, Sierra Club Books. 
LOTHIAN, William F. (1987) Petite histoire des parcs nationaux du Canada. Ottawa, Environnement Canada, Service des parcs.

LOUTER, David (1998) Contested Terrain: North Cascades National Park Service Complex. Seattle, National Park Service.

LOWRY, William R. (1994) The Capacity for Wonder. Preserving National Parks. Washington, The Brooking Institution.

MACHLIS, Gasy E. et TICHNELL, David L. (1985) The State of the World Park. Boulder, Westview.

MAGOC, Chris J. (1999) Yellowstone, the Creation and Selling of an American Landscape, 1870-1903. Albuquerque, The University of New Mexico Press.

MARCHAND, Hélène (2001) Les parcs marins, insulaires et littoraux du Saint-Laurent. Études Canadiennes, vol.27, no50, pp. 101-114.

MARSH, John (1983) Canada's Parks and Tourism: A Problematic Relationship. Peter E. Murphy (dir.) (1983) Tourism in $\mathrm{Ca}$ nada: Selected issues and options, vol.21, Western Geographical Series, Victoria, University of Victoria, pp. 271-307.

MARSH, John P. (1864) Man and Nature, or Physical Geography as Modified by Human Action. New York, C. Scribner and Co.

MOUMANEIX, Caroline (2006a) L'état de la coopération transfrontalière entre les États-Unis et le Canada. Les exemples des parcs frontaliers Wrangell (Alaska)/ Kluane (Yukon) et North Cascades (Washington State)/Manning (ColombieBritannique). Communication au colloque de l'Association Française d'Études Canadiennes, Saumur, 8-10 juin.

MOUMANEIX, Caroline (2006b) Boundaries Around and Within Americain National Parks on the Borderline. Can Wilderness be your Backyard? Communicatin au Colloque de Trento, 12-15 juin.

NASH, Roderick (1982) [1967] Wilderness and the American Mind. New Heaven, Yale University Press.
National Park Service (2000) Everglades National Park Strategic Plan, 2001 - 2005. Floride, National Park Service, US Department of the Interior. [En ligne]. http:// www.nps.gov/ever

National Park Service (2004) Schoodic Draft General Management Plan Amendment and Environmental Impact Statement Acadia National Park - Maine, Northeast Region. National Park Service, US Department of the Interior.

National Park Service (2007) Resources Management Plan: Hot Springs National Park. U.S. Department of the Interior [En ligne]. http://www.nps.gov/hosp/parkmgmt/ upload/RMP97.2.pdf, juin 2007

NELSON, James G., NEEDHAM, Roger D., NELSON, S. H. et SCACE, Robert C. (1979) The Canadian National Parks: Today and Tomorrow, Conference II. Ottawa, James Gordon Nelson.

NEPAL, Sanjay K. (2000) Tourism in Protected Areas: The Nepalese Himalaya. Annals of Tourism Research, vol.27, no3, pp. 661-681.

OELSCHLAEGER, Max (1991) The Idea of Wilderness, From Prehistory to the Age of Ecology. New Haven, Yale University Press.

Parcs Canada (1998) Rapport sur l'état des parcs de 1997. Ottawa, Parcs Canada.

Parcs Canada (2000a) Parc national Jasper du Canada, Plan directeur, mai 2000. Ottawa, Parcs Canada.

Parcs Canada (2000b) Parc national des Lacs Waterton du Canada - Élément du parc international de la Paix Waterton-Glacier, Plan Directeur, mai 2000. Ottawa, Ministre des Travaux publics et Services gouvernementaux.

Parcs Canada (2004) Parc national et réserve de parc national du Canada Kluane. Plan directeur. Ottawa, Ministère des travaux publics et des services.

Parcs Canada (2006) Fréquentation à Parcs Canada, 2000-2001 - 2004-2005. Ottawa, Parcs Canada. 
Park Service (1972) Master Plan, Mount Rainier National Park. Washington.

Parks Canada (1997) Banff National Park-East Gate, Winter 1996/97 Traffic Recalibration Program. Banff, Parks Canada.

PERLA, Bianca et al. (2006) History and Impact of Protected Areas in an International US-Canadian Watershed. Études Canadiennes. Communication au colloque de l'Association Française d'Études Canadiennes, Saumur, 8-10 juin.

PRATO, Tony (2003) Alleviating Multiple Threats to Protected Areas with Adaptive Ecosystem Management. The Case of Waterton-Glacier International Peace Park. The George Wright FORUM, vol.20, no 4 , pp. 41-52.

QUIN, Richard (1997) A Vicious Circle: Automobile and the National Parks. The George Wright Forum. Journal of Cultural and Natural parks and Reserves, Proceedings of the $9^{\text {th }}$ Conference on Research and Resource Management in Parks and on Public Lands.

REID, Dennis (1979) Notre patrie le Canada. Mémoires sur les aspirations nationales des principaux paysagistes de Montréal et de Toronto, 1860-1890. Ottawa, Galerie nationale du Canada.

RICHEZ, Gérard (1991) Aménagement et urbanisation dans les Parcs Nationaux des Rocheuses canadiennes. Études canadiennes, no 31 , pp. 31-48.

SACAREAU, Isabelle (1999) Les transformations d'une haute montagne par le tourisme: le massif des Annapurna dans l'Himalaya du Népal. Annales de Géographie, $\mathrm{n}^{\circ} 605$, pp. 21-45.

SANGUIN, André-Louis (1989) Le parc marin du Saguenay-Saint-Laurent (Québec) : un nouveau concept dans l'histoire des parcs nationaux canadiens. Norois, vol.36, $\mathrm{n}^{\circ} 142$, pp. 137-150.

SANGUIN, André-Louis et GILL, Alison (1991) Le Parc national de Jasper (Alberta) : Gestion et aménagement d'un grand espace récréatif des Rocheuses canadiennes. Revue de géographie alpine, vol.79, nº2, pp. 121-136.
SCHMIDT DI FRIEDBERG, Marcella (2004) L'arca di Noè. Conservazionismo tra natura e cultura. Turin, G. Giappichelli Editore.

SELLARS, Richard W. (1997) Preserving Nature in the National Parks, a History. New Heaven, Yale University Press.

SHIFTAN, Yoram et al. (2006) Demand for Park Shuttle Services - A Stated-Preference Approach. Journal of Transport Geography, vol. 14, pp. 52-59.

TICHNELL, David L., MACHLIS, Gary E. et FAZIO, J. R. (1983) Threats to National Park: A preliminary Survey. Parks - An International Journal for Managers of National Parks, Historic Sites, and Other Protected Areas, vol. 8, no 1, pp. 14-17.

TIMOTHY, Dallen J. (2002) Tourism and International Parks. Dans Richard W. Butler et Stephen W. Boyd (dir.) Tourism and National Parks, Issues and Implications, Chichester, John Wiley and Sons, pp. 263-282.

TUAN, Yi-fu (1990) [1974] Topophilia, A Study of Environmental Perception, Attitudes and Values. Englewood Cliffs, Prentice-Hall.

VÁSÁRHELYI, Charlotte et THOMAS Vernon G. (2006) Evaluating the Capacity of Canadian and American Legislation to Implement Terrestrial Protected Areas Networks. Environmental Science \& Policy, vol.9, $n^{\circ} 1$, pp. 46-54.

VIARD, Jean (1985) Protestante, la nature? Dans Alain Cadoret (dir.) Protection de la nature, histoire et idéologie: de la nature à l'environnement, Paris, L'Harmattan, pp. 161-173.

WORSTER, Donald (1998) [1977] Les pionniers de l'écologie. Paris, Éditions Sang de la Terre. 